\title{
Testing individual baroreflex responses to hypoxia-induced peripheral chemoreflex stimulation
}

\author{
Hendrik Kronsbein ${ }^{1,2}$. Darius A. Gerlach ${ }^{1}$ (1) Karsten Heusser ${ }^{1} \cdot$ Alex Hoff $^{1} \cdot$ Fabian Hoffmann $^{1,3}$. André Diedrich ${ }^{4}$. \\ Heimo Ehmke ${ }^{2,5} \cdot$ Jens Jordan ${ }^{6} \cdot$ Jens Tank ${ }^{1}$
}

Received: 13 September 2019 / Accepted: 21 December 2019 / Published online: 23 January 2020

(c) The Author(s) 2020

\begin{abstract}
Introduction Baroreflexes and peripheral chemoreflexes control efferent autonomic activity making these reflexes treatment targets for arterial hypertension. The literature on their interaction is controversial, with suggestions that their individual and collective influence on blood pressure and heart rate regulation is variable. Therefore, we applied a study design that allows the elucidation of individual baroreflex-chemoreflex interactions.

Methods We studied nine healthy young men who breathed either normal air (normoxia) or an air-nitrogen-carbon dioxide mixture with decreased oxygen content (hypoxia) for $90 \mathrm{~min}$, with randomization to condition, followed by a 30-min recovery period and then exposure to the other condition for $90 \mathrm{~min}$. Multiple intravenous phenylephrine bolus doses were applied per condition to determine phenylephrine pressor sensitivity as an estimate of baroreflex blood pressure buffering and cardiovagal baroreflex sensitivity (BRS).

Results Hypoxia reduced arterial oxygen saturation from $98.1 \pm 0.4$ to $81.0 \pm 0.4 \%(p<0.001)$, raised heart rate from $62.9 \pm 2.1$ to $76.0 \pm 3.6 \mathrm{bpm}(p<0.001)$, but did not change systolic blood pressure $(p=0.182)$. Of the nine subjects, six had significantly lower BRS in hypoxia $(p<0.05)$, two showed a significantly decreased pressor response, and three showed a significantly increased pressor response to phenylephrine in hypoxia, likely through reduced baroreflex buffering $(p<0.05)$. On average, hypoxia decreased BRS by $6.4 \pm 0.9 \mathrm{~ms} / \mathrm{mmHg}(19.9 \pm 2.0$ vs. $14.12 \pm 1.6 \mathrm{~ms} / \mathrm{mmHg} ; p<0.001)$ but did not change the phenylephrine pressor response $(p=0.878)$.

Conclusion We applied an approach to assess individual baroreflex-chemoreflex interactions in human subjects. A subgroup exhibited significant impairments in baroreflex blood pressure buffering and BRS with peripheral chemoreflex activation. The methodology may have utility in elucidating individual pathophysiology and in targeting treatments modulating baroreflex or chemoreflex function.
\end{abstract}

Keywords Cardiovascular $\cdot$ Blood pressure $\cdot$ Pharmacological baroreflex $\cdot$ Chemoreflex $\cdot$ Phenylephrine

H. Kronsbein and D. A. Gerlach contributed equally to this work.

Jens Tank

jens.tank@dlr.de

1 Department of Cardiovascular Aerospace Medicine, Institute of Aerospace Medicine, German Aerospace Center (DLR), 51147 Cologne, Germany

2 Institute of Cellular and Integrative Physiology, University Medical Center Hamburg-Eppendorf, Hamburg, Germany

3 Division of Cardiology, Angiology and Pneumology, University Hospital Cologne, Cologne, Germany
4 Division of Medicine, Division of Clinical Pharmacology, Autonomic Dysfunction Service, Vanderbilt University, Nashville, TN, USA

5 Deutsches Zentrum für Herz-Kreislaufforschung (German Centre for Cardiovascular Research), Hamburg-Kiel-Lübeck, Germany

6 Institute of Aerospace Medicine, German Aerospace Center (DLR) and Chair of Aerospace Medicine, Cologne, Germany 


\section{Introduction}

Arterial baroreflexes and peripheral chemoreflexes have been implicated in the pathogenesis of arterial hypertension and identified as treatment targets [1]. Both originate from receptors, namely, the carotid sinus baroreceptors and carotid body chemoreceptors, respectively, located in close proximity to the carotid bifurcation [2,3]. Baroreflexes buffer changes in blood pressure through counterregulatory adjustments in efferent sympathetic and parasympathetic activity and affect long-term blood pressure control [4], and carotid body chemoreceptor stimulation raises sympathetic activity [5]. In arterial hypertension, the sympathetic baroreflex gain is depressed and the baroreflex is reset to higher blood pressure [6]; in contrast, carotid chemoreceptors are sensitized [7-9] and generate tonic sympathetic activation [10-13] in hypertensive patients. Baroreceptor and carotid body afferents converge onto neurons in the dorsomedial medulla [14], resulting in an inhibitory interaction $[15,16]$. Thus, impaired baroreflex regulation in arterial hypertension may be mediated in part through inappropriate carotid body chemoreceptor activation $[1,9]$. Electrical carotid sinus stimulators and stents augmenting baroreceptor transmission as well as peripheral chemoreceptor modulation have been developed for hypertension management; however, all baroreceptor reflex modulation procedures developed to date exhibit an unacceptably high proportion of non-responders [17-20]. Blood pressure reductions following such interventions are likely determined by interindividual differences in baroreflex-chemoreflex interactions, which are not uncovered through routine clinical testing. While human baroreflex-chemoreflex interactions have been previously investigated [16, 21, 22], we hypothesized that a combination of physiological profiling and multiple repeated measurements could be utilized to elucidate individual baroreflex-chemoreflex interactions. In our study, hypoxia served as the peripheral chemoreceptor stimulus. In a similar fashion, patients have been repeatedly exposed to test and control interventions, such as statin and placebo treatment, to elucidate individual responses in so-called $\mathrm{N}$-ofone trials $[23,24]$.

\section{Methods}

\section{Subjects}

Nine healthy young men with a mean age of $28.8 \pm 3.0$ years and a mean body mass index of $23.8 \pm 0.8 \mathrm{~kg} / \mathrm{m}^{2}$ were enrolled in the study (Table 1). Medical history and results from the physical examination, resting electrocardiogram, spirometry, and routine blood testing were all in the normal range. The ethics committee of the North Rhine medical association approved the study, and the study was registered in the German Clinical Trials register (DRKS00013101). Written informed consent was obtained from each participant before study entry.

\section{Protocol}

All measurements were obtained with the subjects in the supine position after they had voided their bladder. We assessed non-invasive beat-to-beat blood pressure (Finapres ${ }^{\circledR}$ NOVA; Finapres Medical Systems, Enschede, The Netherlands), upper arm blood pressure, arterial oxygen saturation, end-tidal carbon dioxide $\left(\mathrm{ETCO}_{2}\right)$ concentration, breathing frequency, tidal volume, minute ventilation (Innocor; Innovision, Odense, Denmark), and an electrocardiogram recording (Vital Guard 450C; Ivy Biomedical Systems, Inc., Branford, CT, USA). An antecubital venous catheter was set in place for drug administration. Baseline data were recorded for $10 \mathrm{~min}$ prior to starting the randomized protocol (Table 1).
Table 1 Baseline characteristics of subjects $(n=9)$

\begin{tabular}{llllll}
\hline Subject & SBP $(\mathrm{mmHg})$ & DBP $(\mathrm{mmHg})$ & HR $(\mathrm{bpm})$ & BMI $\left(\mathrm{kg} / \mathrm{m}^{2}\right)$ & Age (years) \\
\hline 01 & 126 & 78 & 61 & 27.4 & 37 \\
02 & 136 & 78 & 61 & 24.5 & 30 \\
03 & 122 & 74 & 65 & 22.6 & 24 \\
04 & 132 & 82 & 66 & 24.5 & 38 \\
05 & 136 & 70 & 67 & 24.2 & 20 \\
06 & 125 & 80 & 79 & 21.2 & 22 \\
07 & 139 & 82 & 48 & 24.3 & 34 \\
08 & 127 & 62 & 80 & 20.8 & 24 \\
09 & 127 & 72 & 55 & 22.6 & 33 \\
\hline
\end{tabular}

Values in table are the baseline characteristics of each subject taken before the beginning of the first session $S B P$ Systolic blood pressure, $D B P$ diastolic blood pressure, $H R$ heart rate, $B M I$ body mass index 
We first determined the individual dose of the alpha1 -adrenoreceptor agonist phenylephrine that was needed to raise to blood pressure by $20-30 \mathrm{mmHg}$, using incremental doses of between 25 and $100 \mu \mathrm{g}$. All phenylephrine doses were immediately followed by a $10-\mathrm{ml}$ saline flush. Both procedures were conducted in a standardized fashion using an automated bolus injector (Accutron MR; Medtron AG, Saarbrücken, Germany). Bolus flow rates were $5 \mathrm{ml} / \mathrm{s}$ for phenylephrine and $2.5 \mathrm{ml} / \mathrm{s}$ for the saline bolus, resulting in administration durations of 4.1-4.4 s. Following determination of the appropriate dose, we obtained repeated baroreflex measurements using the predetermined phenylephrine dose, with the subjects exposed to either the normoxic or hypoxic condition for $90 \mathrm{~min}$, with randomization to one or the other condition and single blinding (subjects). Following the first exposure, subjects were allowed to recover for 30 min during which time they could stand up and empty their bladder, with the aim to minimize any carry-over effects from being supine or hypoxic, before being exposed for $90 \mathrm{~min}$ to the other condition. In the first $10 \mathrm{~min}$ of each exposure, inspiratory oxygen was slowly lowered to attain hypoxia or kept constant for normoxia testing. Thereafter, 20 phenylephrine bolus doses were applied, one every $4 \mathrm{~min}$. The study protocol is illustrated in Fig. 1.

\section{Hypoxic peripheral chemoreflex stimulation}

We utilized a hypoxic gas mixture containing nitrogen, oxygen, and carbon dioxide. Compressed air and nitrogen were mixed in a blender (Bird ${ }^{\mathrm{TM}}$ Blender low flow; Vyaire Medical Inc., Mettawa, IL, USA). Carbon dioxide was added separately in order to maintain normocapnia. Gas flows were controlled using flowmeters and buffered in a Douglas bag from which the subject could inhale without resistance or pressure via a face mask. For normocapnic hypoxia, the target was $80 \%$ arterial oxygen saturation, achieved by manual adjustment. To keep subjects blind to the condition, the same toolset was used to apply compressed air during both the hypoxia and normoxia procedures.

\section{Baroreflex measurements}

Two parameters that characterize baroreflex function were assessed, namely, cardiovagal baroreflex sensitivity (BRS) and baroreflex buffering capacity. For both measurements we utilized the increase in finger blood pressure after the administration of each phenylephrine bolus. This pharmacological approach ensures greater independence from ventilatory influences on baroreflex function than methods based on spontaneous variations in hemodynamic variables. The investigator was blinded to the condition, i.e., normoxia or hypoxia.

1. Cardiovagal BRS was assessed as the slope of the regression line between each systolic blood pressure (SBP) value and the following RR interval during the phenylephrine-induced pressure rise without an offset $[25,26]$. The slope was accepted if Pearson's correlation coefficient $r>0.5$ [27].

2. Larger increases in blood pressure after the administration of a defined phenylephrine bolus denote reduced the baroreflex buffering capacity. We determined this phenylephrine sensitivity as rise in $\mathrm{SBP}(\triangle \mathrm{SBP})$ from the baseline before each bolus to the SBP maximum after bolus application [28]. Baseline SBP was calculated by averaging ten readings taken before each bolus. Maximum SBP was obtained by averaging the three highest consecutive SBP values within $60 \mathrm{~s}$ after each bolus.

\section{Statistics}

Individual BRS and phenylephrine responses were analyzed using unpaired $t$ tests. For group comparisons, we used medians of up to 20 boli per condition in order to minimize the effect of possible outliers and analyzed these via the paired $t$ test. Significance was assumed at $p<0.05$. If not otherwise indicated, all values were expressed as the mean \pm SE. Power for BRS and change in SBP were
Fig. 1 Schematic illustration of the study protocol. Phe Phenylephrine
Protocol

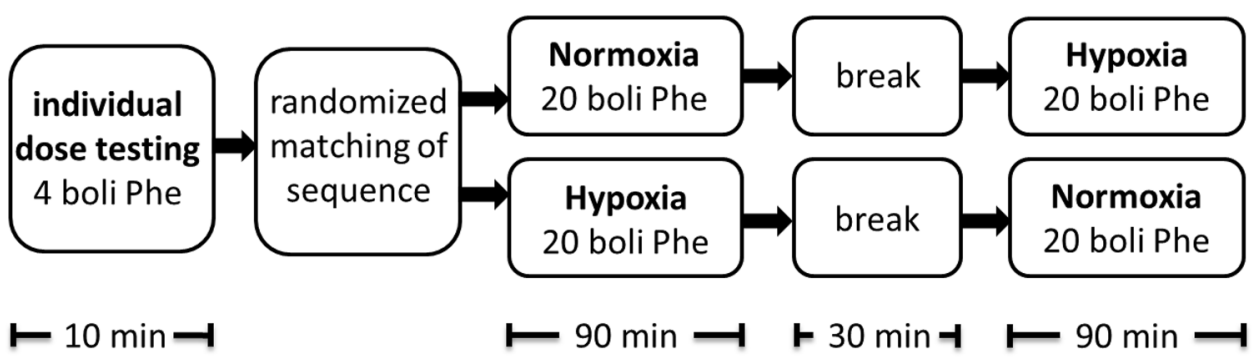


calculated post hoc by means of the statistical analysis tool $\mathrm{G}^{*}$ Power 3.1. We estimated the number of bolus repetitions needed to estimate individual effects between normoxia and hypoxia with receiver operating characteristic curve analysis performed with SPSS V.21 software (IBM Corp., Armonk, NY, USA). All other statistical analyses were done using Graphpad Prism 7.04 (GraphPad Software Inc., San Diego, CA, USA)

\section{Results}

All subjects tolerated baroreflex testing in both normoxia and hypoxia, and no adverse events were observed. Arterial oxygen saturation was $98.1 \pm 0.4 \%$ during normoxia and $81.0 \pm 0.4 \%$ during hypoxia $(p<0.001)$, and $\mathrm{ETCO}_{2}$ remained at baseline values $(38.31 \pm 0.9$ [normoxia] vs. $37.78 \pm 1.1 \mathrm{mmHg}$ [hypoxia]; $p=0.245$ ). Respiratory rate $(16 \pm 1$ vs. $16 \pm 1 / \mathrm{min} ; p=0.588)$ did not change. During hypoxia, tidal volume increased from $0.77 \pm 0.04$ to $0.95 \pm 0.071(p=0.002)$, and minute ventilation increased from $11.86 \pm 0.51$ to $13.95 \pm 0.49 \mathrm{l} / \mathrm{min}(p=0.036)$; the mean respiratory chemoreflex response $\left(\Delta \mathrm{Ve} / \mathrm{SpO}_{2}\right)$ was $11.79 \pm 4.80(1 / \mathrm{min}) / \%$.

Blood pressure was $133 \pm 3 / 76 \pm 2 \mathrm{mmHg}$ during normoxia and $135 \pm 3 / 76 \pm 2 \mathrm{mmHg}$ during hypoxia. During the experiment systolic blood pressure ranged from 122 to $154 \mathrm{mmHg}$ during normoxia and from 121 to $148 \mathrm{mmHg}$ during hypoxia. Heart rate increased from $63 \pm 2 \mathrm{bpm}$ during normoxia to $76 \pm 4 \mathrm{bpm}$ during hypoxia $(p<0.001)$. Figure 2 illustrates original blood pressure and RR-interval responses to repeated phenylephrine doses during normoxia and during hypoxia in one subject.

Individual baroreflex responses are given in Tables 2, 3, and Fig. 3. Not all phenylephrine applications yielded responses suitable for baroreflex analysis. The number of bolus doses analyzed for each subject and condition ranged between 8 and 20 (Tables 2, 3). In hypoxia, BRS was significantly lowered in six subjects; Figure 3 a illustrates individual changes in BRS, including the $95 \%$ confidence intervals, with reductions in BRS during hypoxia in these six subjects. Overall BRS decreased from $19.9 \pm 2.0 \mathrm{~ms} / \mathrm{mmHg}$ in normoxia to $14.1 \pm 1.6 \mathrm{~ms} / \mathrm{mmHg}$ in hypoxia $(p<0.001$; power 0.995; Fig. 3a).

Five subjects showed a significant change in phenylephrine responsiveness with hypoxic peripheral chemoreflex stimulation (Table 3), with phenylephrine responsiveness significantly decreasing in two subjects and significantly increasing in three subjects. In the other four subjects, phenylephrine sensitivity did not change significantly. Overall, phenylephrine responsiveness did not change $(p=0.878)$, as shown in Fig. 3b, which plots individual changes in phenylephrine sensitivity, including the $95 \%$ confidence intervals, and indicates the high interindividual variability.

The optimal number of repetitions for individual BRS changes was ten boli with a sensitivity of 0.96. This is derived from our heathy subjects with an effect size of $6 \pm 3 \mathrm{~ms} / \mathrm{mmHg}$ (difference in BRS between normoxia and hypoxia).Overall, we did not observe a linear correlation between changes in BRS or phenylephrine responsiveness and respiratory parameters (respiratory rate, tidal volume, or minute ventilation). Individual respiratory parameters varied, as shown in Table 4.

Hemodynamic reactions were stable over time during normoxia and hypoxia (Fig. 2). The sequence of the conditions normoxia and hypoxia did not affect BRS or phenylephrine responsiveness measurements.

We could not find a correlation between changes in cardiac BRS and baroreflex buffering capacity (Fig. 4).

\section{Discussion}

In the study reported here, we applied a novel approach to elucidate individual baroreflex-chemoreflex interactions using repeated administrations of a standardized phenylephrine bolus during normoxia and hypoxia. While individual and group cardiovagal BRS responses to hypoxic peripheral chemoreceptor stimulation showed similar qualitative responses, individual responses exhibited substantial quantitative variability. Strikingly, individual phenylephrine responsiveness revealed qualitatively different responses, possibly resulting from changes in vascular sensitivity, in baroreflex blood pressure buffering, or in both mechanisms combined [28]. Our findings provide insight into human autonomic cardiovascular control mechanisms and could be useful in individualized assessment of baroreflex-chemoreflex interactions in patients with arterial hypertension, particularly in those considered for interventions targeting these reflexes.

The highly standardized assessment of baroreflex function using phenylephrine doses applied through an automated injector is a particular strength of our study. In previous studies, vasoactive drugs for baroreflex testing as well as subsequent saline flushes were manually applied [21, 29]. The lack of standardization likely introduces variability in drug delivery, immediate vascular responses, and subsequent baroreflex counter regulation. Our approach reduced this variability source. Another strength of our study is the large number of repeated phenylephrine tests which, unlike in previous studies, allowed for individual assessment of the baroreflex-chemoreflex interaction. We found that ten boli with a significant cardiovagal baroreflex response could be the appropriate number of boli for an optimal estimation 

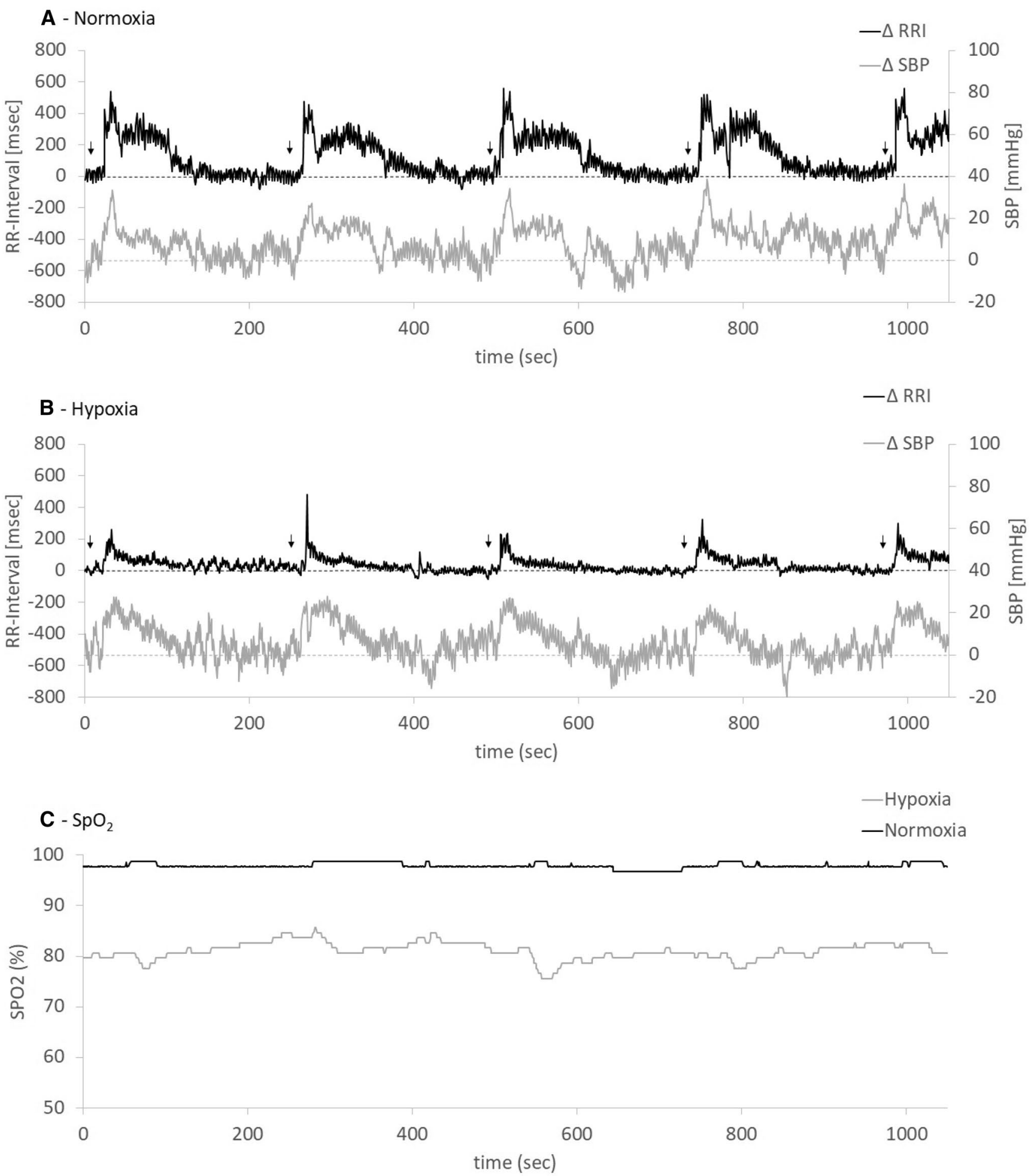

Fig. 2 Original recording of RR-interval $(R R I)$ and systolic blood pressure $(S B P)$ responses to phenylephrine boli given during normoxia (a) and hypoxia (b), as well as corresponding peripheral capillary oxygen saturation $(\mathrm{SpO} 2)$ curves $(\mathbf{c})$. a, b Black arrows show timepoints at which phenylephrine boli were injected; the black upper lines correspond to RRI with the RRI axis on the left, and the lower gray lines correspond to SBP with the axis on the right side 
Table 2 Individual baroreflex responses of the nine subjects in normoxia and hypoxia

\begin{tabular}{|c|c|c|c|c|c|c|c|}
\hline \multirow[t]{2}{*}{ Subject } & \multirow{2}{*}{$\begin{array}{l}\text { Order of condition applied } \\
\text { (condition applied first) }\end{array}$} & \multicolumn{2}{|l|}{ Normoxia } & \multicolumn{2}{|l|}{ Hypoxia } & \multicolumn{2}{|c|}{ Statistical analysis } \\
\hline & & $\mathrm{BRS}(\mathrm{ms} / \mathrm{mmHg})$ & Boli $(n)$ & $\mathrm{BRS}(\mathrm{ms} / \mathrm{mmHg})$ & boli $(n)$ & $p$ value & $t$ \\
\hline 01 & Hypoxia & $19.6 \pm 2.1$ & 17 & $18.3 \pm 1.4$ & 19 & 0.603 & 0.525 \\
\hline 02 & Normoxia & $14.9 \pm 1.4$ & 16 & $9.5 \pm 1.4$ & 17 & 0.011 & 2.689 \\
\hline 03 & Hypoxia & $18.9 \pm 1.9$ & 18 & $11.4 \pm 1.2$ & 17 & 0.002 & 3.307 \\
\hline 04 & Normoxia & $14.4 \pm 1.2$ & 19 & $11.1 \pm 2.1$ & 17 & 0.159 & 1.439 \\
\hline 05 & Normoxia & $29.0 \pm 2.0$ & 14 & $19.6 \pm 1.9$ & 16 & 0.002 & 3.381 \\
\hline 06 & Hypoxia & $14.7 \pm 1.2$ & 16 & $10.1 \pm 0.9$ & 17 & 0.017 & 2.533 \\
\hline 07 & Normoxia & $28.6 \pm 3.3$ & 16 & $16.8 \pm 2.6$ & 8 & 0.028 & 2.35 \\
\hline 08 & Hypoxia & $14.4 \pm 0.8$ & 20 & $8.3 \pm 0.8$ & 20 & $<0.001$ & 5.399 \\
\hline 09 & Hypoxia & $24.9 \pm 1.6$ & 19 & $21.1 \pm 1.8$ & 17 & 0.122 & 1.585 \\
\hline
\end{tabular}

$B R S$ Baroreflex sensitivity

BRS values are presented as the individual mean values \pm standard error of the mean (SEM), together with the corresponding number of boli $p$ values and $t$ correspond to measurements in normoxia vs. those in hypoxia. Significance was assumed at $p<0.05$

Table 3 Change in systolic blood pressure in normoxia and hypoxia in the nine subjects

\begin{tabular}{|c|c|c|c|c|c|c|c|}
\hline \multirow[t]{2}{*}{ Subject } & \multirow{2}{*}{$\begin{array}{l}\text { Order of condition } \\
\text { applied (condition } \\
\text { applied first) }\end{array}$} & \multicolumn{2}{|l|}{ Normoxia } & \multicolumn{2}{|l|}{ Hypoxia } & \multicolumn{2}{|c|}{$\begin{array}{l}\text { Statistical } \\
\text { analysis }\end{array}$} \\
\hline & & $\Delta \mathrm{SBP}(\mathrm{mmHg})$ & $\overline{\operatorname{Boli}(n)}$ & $\Delta \mathrm{SBP}(\mathrm{mmHg})$ & $\overline{\text { Boli }(n)}$ & $p$ value & $t$ \\
\hline 01 & Hypoxia & $17.6 \pm 1.2$ & 20 & $21.8 \pm 1.4$ & 20 & 0.026 & 2.31 \\
\hline 02 & Normoxia & $25.7 \pm 2.7$ & 20 & $23.2 \pm 2.9$ & 17 & 0.531 & 0.633 \\
\hline 03 & Hypoxia & $27.8 \pm 1.1$ & 20 & $31.0 \pm 2.4$ & 20 & 0.232 & 1.214 \\
\hline 04 & Normoxia & $19.4 \pm 1.7$ & 19 & $29.7 \pm 2.3$ & 18 & 0.001 & 3.576 \\
\hline 05 & Normoxia & $18.1 \pm 1.6$ & 19 & $24.1 \pm 2.2$ & 20 & 0.033 & 2.21 \\
\hline 06 & Hypoxia & $30.0 \pm 1.7$ & 20 & $26.0 \pm 2.0$ & 20 & 0.1396 & 1.509 \\
\hline 07 & Normoxia & $17.1 \pm 1.8$ & 20 & $13.0 \pm 1.4$ & 20 & 0.069 & 1.872 \\
\hline 08 & Hypoxia & $30.3 \pm 1.3$ & 20 & $22.2 \pm 1.1$ & 20 & $<0.001$ & 4.714 \\
\hline 09 & Hypoxia & $19.1 \pm 1.2$ & 20 & $12.5 \pm 1.9$ & 20 & 0.006 & 2.943 \\
\hline
\end{tabular}

$\Delta$ SBP values are presented as individual mean values \pm SEM, together with the corresponding number of boli

$p$ values and $t$ correspond to measurements in normoxia vs. those in hypoxia. Significance was assumed at $p<0.05$
Fig. 3 Forest plots of individual changes (hypoxia-normoxia) in cardiovagal baroreflex sensitivity $(B R S)(\mathbf{a})$ and individual changes in phenylephrine sensitivity (b). Data points indicate mean difference in BRS or SBP increase between hypoxia and normoxia, and error bars indicate the range of these differences $(95 \%$ confidence interval). $p$ values are shown next to each data point, and asterisks, where present, indicate significance of difference $(* p<0.05$; $* * p<0.01 ; * * * p<0.001)$

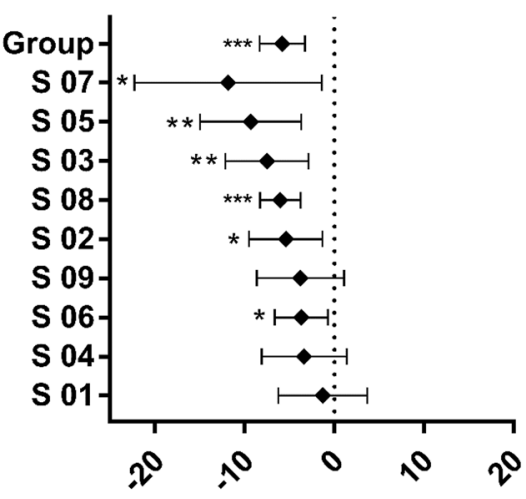

3A $\triangle$ BRS [msec/mmHg]

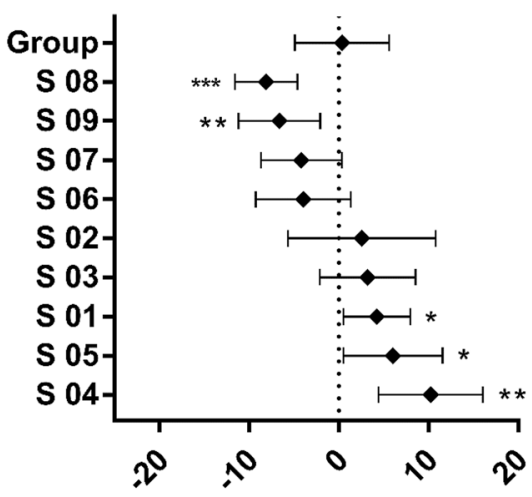

3B 
Table 4 Respiration parameters recorded for each of the nine subjects throughout each session in normoxia or hypoxia

\begin{tabular}{|c|c|c|c|c|c|c|c|c|}
\hline \multirow[t]{2}{*}{ Subject } & \multicolumn{2}{|c|}{ Respiratory rate (breaths/min) } & \multicolumn{2}{|c|}{ Tidal volume (l) } & \multicolumn{2}{|c|}{ Minute ventilation (1/min) } & \multirow[t]{2}{*}{$\Delta \mathrm{SpO}_{2}(\%)$} & \multirow{2}{*}{$\begin{array}{l}\Delta \mathrm{Ve} / \\
\Delta \mathrm{SpO}_{22}([1 / \\
\min ] / \%)\end{array}$} \\
\hline & Normoxia & Hypoxia & Normoxia & Hypoxia & Normoxia & Hypoxia & & \\
\hline 01 & $9.53 \pm 0.10$ & $13.04 \pm 0.08$ & $1.05 \pm 0.02$ & $1.33 \pm 0.01$ & $9.33 \pm 0.12$ & $16.86 \pm 0.11$ & 17.30 & 43.53 \\
\hline 02 & $17.11 \pm 0.05$ & $17.95 \pm 0.13$ & $0.80 \pm 0.00$ & $0.88 \pm 0.01$ & $13.64 \pm 0.06$ & $15.67 \pm 0.15$ & 18.51 & 11.11 \\
\hline 03 & $14.13 \pm 0.11$ & $12.55 \pm 0.16$ & $0.87 \pm 0.01$ & $1.13 \pm 0.02$ & $11.62 \pm 0.09$ & $12.48 \pm 0.14$ & 16.53 & 5.25 \\
\hline 04 & $16.30 \pm 0.09$ & $16.02 \pm 0.09$ & $0.73 \pm 0.01$ & $0.83 \pm 0.01$ & $11.64 \pm 0.05$ & $12.77 \pm 0.10$ & 16.40 & 6.91 \\
\hline 05 & $19.12 \pm 0.08$ & $20.26 \pm 0.08$ & $0.69 \pm 0.01$ & $0.73 \pm 0.01$ & $12.53 \pm 0.07$ & $14.13 \pm 0.08$ & 16.86 & 9.48 \\
\hline 06 & $19.13 \pm 0.08$ & $17.36 \pm 0.12$ & $0.73 \pm 0.01$ & $0.76 \pm 0.01$ & $13.66 \pm 0.05$ & $12.50 \pm 0.09$ & 15.29 & -7.57 \\
\hline 07 & $18.89 \pm 0.09$ & $14.09 \pm 0.13$ & $0.74 \pm 0.01$ & $1.04 \pm 0.02$ & $13.17 \pm 0.08$ & $13.52 \pm 0.20$ & 16.89 & 2.05 \\
\hline 08 & $18.29 \pm 0.07$ & $18.3 \pm 0.09$ & $0.62 \pm 0.00$ & $0.77 \pm 0.00$ & $11.13 \pm 0.03$ & $13.64 \pm 0.05$ & 19.03 & 13.20 \\
\hline 09 & $14.15 \pm 0.08$ & $13.19 \pm 0.08$ & $0.72 \pm 0.00$ & $1.07 \pm 0.01$ & $10.02 \pm 0.06$ & $13.94 \pm 0.08$ & 17.74 & 22.11 \\
\hline
\end{tabular}

$\mathrm{Ve}$ Minute ventilation, $\mathrm{SpO}_{2}$ peripheral capillary oxygen saturation, $\Delta \mathrm{Vel} \Delta \mathrm{SpO}_{2}$ mean respiratory chemoreflex response Where appropriate, values are given as the mean \pm SEM

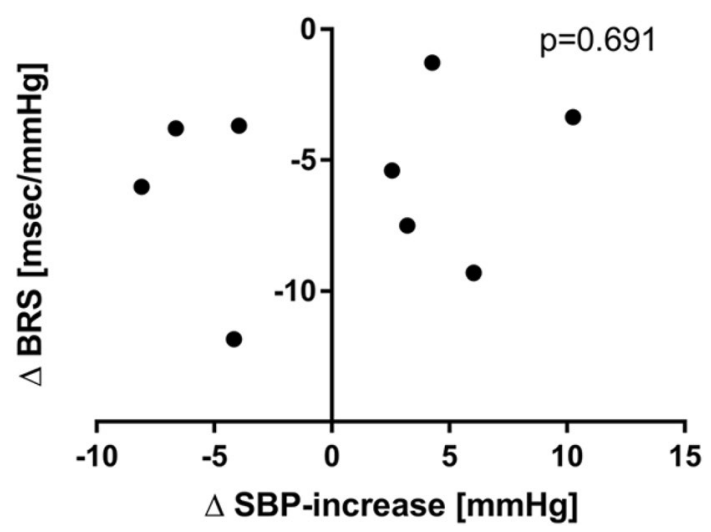

Fig. 4 Correlation analysis between changes in cardiovagal baroreflex sensitivity and phenylephrine sensitivity. Phenylephrine sensitivity is considered as a marker of baroreflex-mediated buffering of blood pressure

of individual BRS changes induced by hypoxic peripheral chemoreceptor activation in the clinical setting.

We observed complex changes in baroreflex regulation with hypoxic peripheral chemoreflex stimulation. Average blood pressure was unchanged while the heart rate increased substantially. The baroreflex heart rate/pressure relationship was shifted towards higher heart rates and cardiovagal BRS was reduced. Increases in heart rate and maintained or increased blood pressure with peripheral chemoreflex stimulation have been previously described; in these studies sympathetic and parasympathetic baroreflex gains $[21,22$, $30,31]$ and baroreflex responsiveness [32] did not change. However, in two studies, chemoreflex-induced resetting of baroreflex-mediated sympathetic vasomotor control to higher blood pressure levels was observed using spontaneous baroreflex threshold [21] and modified Oxford techniques [29]. We speculate that the discrepancy in cardiovagal BRS responses may result in part from the methodology to assess baroreflex function or the high variability which we reduced through repeated testing. Interindividual and between-study variability in the BRS response to hypoxic chemoreceptor stimulation may partly result from variability in chemosensitivity [33].

Baroreflex regulation of sympathetic traffic to the vasculature is particularly relevant for blood pressure control. However, direct sympathetic nerve recordings are available in only a few medical centers and the tests often fail for technical reasons. As an alternative, the primary function of the baroreflex, which is to buffer blood pressure changes, can be assessed by applying vasoactive drugs before and during complete interruption of the baroreflex arc through ganglionic blockade [28]; the sensitivity to vasoactive drugs changes less in individuals with less efficient baroreflex blood pressure buffering than in those with more efficient baroreflex blood pressure buffering. Influences of gender, age, autonomic failure, and arterial hypertension on blood pressure buffering have been previously assessed [34-36]. Ganglionic blockers are no longer available for investigations on humans, and the methodology is cumbersome. Approximately $80 \%$ of the variability in systemic phenylephrine pressor sensitivity can be attributed to variability in baroreflex buffering rather than to vascular responsiveness [28]. That analysis included healthy persons and patients with various disorders known to affect vascular alphaadrenoreceptor responsiveness. Therefore, phenylephrine responses without ganglionic blockade may provide an estimate of baroreflex buffering capacity. Thus, the results of our study may suggest that there is a dissociation between cardiovagal BRS and baroreflex blood pressure buffering during hypoxic chemoreflex stimulation.

The major limitation of our study is that findings in healthy subjects cannot be simply extrapolated to patients 
with arterial hypertension, particularly those considered for device-based treatments targeting the baroreflex or peripheral chemoreceptors. The fact that we did not assess sympathetic nerve activity is another potential limitation. Moreover, we determined an estimate of baroreflex blood pressure buffering rather than making a comparison phenylephrine responses before and after ganglionic blockade for the reasons mentioned above. While much of the interindividual variability in phenylephrine responsiveness can be explained by baroreflex blood pressure buffering, hypoxia-induced changes in vascular alpha-adrenoreceptor responsiveness may have confounded the analysis. However, data from human [37] and animal studies [38] regarding direct vascular actions of hypoxia are controversial, and it is evident that changes in vascular tone during hypoxia cannot be solely explained by baroreflexmediated changes in adrenergic drive. Our observed BRS and phenylephrine responsiveness shift with hypoxia may be the result of hypoxia-induced vasodilation [39, 40]. Furthermore, hypoxia-induced heart rate changes are not abolished following bilateral carotid body removal [41]. However, in patients with resistant arterial hypertension, reductions in sympathetic traffic and blood pressure with electrical carotid sinus stimulation were found to be virtually identical with and without hypoxia [42]. This observation excludes major changes in the coupling between sympathetic activity and vasoconstriction with hypoxia. $\mathrm{ETCO}_{2}$, which affects cardiovascular control, was kept constant during our study. While we applied hypoxia in a steady-state fashion, transient hypoxia has been proposed to provide a more selective peripheral chemoreceptor stimulus [43]. Finally, hypoxia may have a prolonged effect on sympathetic activity [44] - although the sequence of normoxia and hypoxia sessions in our study did not affect our results. Moreover, we did not observe a substantial sustained effect of hypoxia on sympathetic activity or the response to electrical carotid sinus stimulation in patients with treatment-resistant arterial hypertension [42].

Despite these limitations, our study suggests that interindividual variability of baroreflex and chemoreflex regulation is so high that treatments modulating these mechanisms are exceedingly unlikely to be equally effective in all patients. In recent years there has been a thrust towards precision medicine [45]. Usually, the term implicates molecular assessment of a disease trait, such as somatic mutations in a specific cancer, that results in a targeted intervention. Outside oncology, the merits of the approach in common conditions, including arterial hypertension, have been limited; indeed, molecular signatures guiding hypertension treatment do not exist. However, individual physiological profiling could be utilized to identify underlying mechanisms driving hypertension and to direct treatments to patients more likely to respond. Our study suggests that repeated phenylephrine testing during normoxia and during hypoxia deserves to be tested in this setting.

Acknowledgements Open Access funding provided by Projekt DEAL. We thank the volunteers for participating in this study and Christine Becker for the support during the recruitment process. We are grateful to Dr. Bernd Johannes for the statistics consultation.

Funding FH was supported by DLR grant 50WB1816.

Data availability The raw data supporting the conclusions of this manuscript are available at the following link: https://figshare.com/ $\mathrm{s} / 5 \mathrm{fbc} 8 \mathrm{cff} 9925 \mathrm{cec} 758 \mathrm{~d} 4$. This includes the raw analog sampled data from beat-to-beat blood pressure, arterial oxygen saturation, $\mathrm{ETCO}_{2}$ concentration, breathing frequency, tidal volume, minute ventilation, and an electrocardiogram.

\section{Compliance with ethical standards}

Conflict of interest The authors declare that they do not have any conflict of interest.

Open Access This article is licensed under a Creative Commons Attribution 4.0 International License, which permits use, sharing, adaptation, distribution and reproduction in any medium or format, as long as you give appropriate credit to the original author(s) and the source, provide a link to the Creative Commons licence, and indicate if changes were made. The images or other third party material in this article are included in the article's Creative Commons licence, unless indicated otherwise in a credit line to the material. If material is not included in the article's Creative Commons licence and your intended use is not permitted by statutory regulation or exceeds the permitted use, you will need to obtain permission directly from the copyright holder. To view a copy of this licence, visit http://creativecommons.org/licenses/by/4.0/.

\section{References}

1. Paton JF, Sobotka PA, Fudim M, Engelman ZJ, Hart EC, McBryde FD, Abdala AP, Marina N, Gourine AV, Lobo M, Patel N, Burchell A, Ratcliffe L, Nightingale A (2013) The carotid body as a therapeutic target for the treatment of sympathetically mediated diseases. Hypertension 61(1):5-13

2. Lindsey BG, Nuding SC, Segers LS, Morris KF (2018) Carotid bodies and the integrated cardiorespiratory response to hypoxia. Physiology (Bethesda). 33(4):281-297

3. Benarroch EE (2008) The arterial baroreflex: functional organization and involvement in neurologic disease. Neurology 71(21):1733-1738

4. Lohmeier TE, Iliescu R (2015) The baroreflex as a long-term controller of arterial pressure. Physiology (Bethesda) 30(2):148-158

5. Saito M, Mano T, Iwase S, Koga K, Abe H, Yamazaki Y (1988) Responses in muscle sympathetic activity to acute hypoxia in humans. J Appl Physiol 65(4):1548-1552

6. Eckberg DL (1979) Carotid baroreflex function in young men with borderline blood pressure elevation. Circulation 59(4):632-636

7. Somers VK, Mark AL, Abboud FM (1988) Potentiation of sympathetic nerve responses to hypoxia in borderline hypertensive subjects. Hypertension 11(6 Pt 2):608-612

8. Trzebski A, Tafil M, Zoltowski M, Przybylski J (1982) Increased sensitivity of the arterial chemoreceptor drive in young men with mild hypertension. Cardiovasc Res 16(3):163-172 
9. McBryde FD, Abdala AP, Hendy EB, Pijacka W, Marvar P, Moraes DJ, Sobotka PA, Paton JF (2013) The carotid body as a putative therapeutic target for the treatment of neurogenic hypertension. Nat Commun 4:2395

10. Abdala AP, McBryde FD, Marina N, Hendy EB, Engelman ZJ, Fudim M, Sobotka PA, Gourine AV, Paton JF (2012) Hypertension is critically dependent on the carotid body input in the spontaneously hypertensive rat. J Physiol 590(17):4269-4277

11. Sinski M, Lewandowski J, Przybylski J, Bidiuk J, Abramczyk P, Ciarka A, Gaciong Z (2012) Tonic activity of carotid body chemoreceptors contributes to the increased sympathetic drive in essential hypertension. Hypertens Res 35(5):487-491

12. Melnikov VN, Divert VE, Komlyagina TG, Consedine NS, Krivoschekov SG (2017) Baseline values of cardiovascular and respiratory parameters predict response to acute hypoxia in young healthy men. Physiol Res 66(3):467-479

13. Pijacka W, Moraes DJ, Ratcliffe LE, Nightingale AK, Hart EC, da Silva MP, Machado BH, McBryde FD, Abdala AP, Ford AP, Paton JF (2016) Purinergic receptors in the carotid body as a new drug target for controlling hypertension. Nat Med 22(10):1151-1159

14. Paton JF, Deuchars J, Li YW, Kasparov S (2001) Properties of solitary tract neurones responding to peripheral arterial chemoreceptors. Neuroscience 105(1):231-248

15. Somers VK, Mark AL, Abboud FM (1991) Interaction of baroreceptor and chemoreceptor reflex control of sympathetic nerve activity in normal humans. J Clin Invest 87(6):1953-1957

16. Cooper VL, Pearson SB, Bowker CM, Elliott MW, Hainsworth R (2005) Interaction of chemoreceptor and baroreceptor reflexes by hypoxia and hypercapnia - a mechanism for promoting hypertension in obstructive sleep apnoea. J Physiol 568(Pt 2):677-687

17. Chunbin W, Fu S, Jing H (2018) Efficacy and safety of baroreflex activation therapy for treatment of resistant hypertension: a systematic review and meta-analysis. Clin Exp Hypertens 40(6):501-508

18. Jordan J, Mann JF, Luft FC (2013) Research needs in the area of device-related treatments for hypertension. Kidney Int 84(2):250-255

19. Wallbach M, Koziolek MJ, Wachter R (2018) Baroreflex activation therapy : Indication and evidence in resistant hypertension and heart failure. Internist (Berl) 59(10):1011-1020

20. Koziolek M, Beige J, Wallbach M, Zenker D, Henning G, Halbach M, Mader N, Mahfoud F, Schlieper G, Schwenger V, Hausberg M, Borgel J, Lodde M, van der Giet M, Muller-Ehmsen J, Passauer J, Parmentier S, Luders S, Kramer BK, Buttner S, Limbourg F, Jordan J, Vonend O, Predel HG, Reuter H (2017) Baroreceptor activation therapy for therapy-resistant hypertension: indications and patient selection: recommendations of the BAT consensus group 2017. Internist (Berl) 58(10):1114-1123

21. Querido JS, Wehrwein EA, Hart EC, Charkoudian N, Henderson WR, Sheel AW (2011) Baroreflex control of muscle sympathetic nerve activity as a mechanism for persistent sympathoexcitation following acute hypoxia in humans. Am J Physiol Regul Integr Comp Physiol 301(6):R1779-1785

22. Halliwill JR, Minson CT (2002) Effect of hypoxia on arterial baroreflex control of heart rate and muscle sympathetic nerve activity in humans. J Appl Physiol 93(3):857-864

23. Ebrahim S, Davey Smith G (2015) N-of-1 approach to determine when adverse effects are caused by statins. BMJ 351:h5281

24. Joy TR, Monjed A, Zou GY, Hegele RA, McDonald CG, Mahon JL (2014) N-of-1 (single-patient) trials for statin-related myalgia. Ann Intern Med 160(5):301-310

25. Smyth HS, Sleight P, Pickering GW (1969) Reflex regulation of arterial pressure during sleep in man. A quantitative method of assessing baroreflex sensitivity. Circ Res 24(1):109-121.
26. Gribbin B, Pickering TG, Sleight P, Peto R (1971) Effect of age and high blood pressure on baroreflex sensitivity in man. Circ Res 29(4):424-431

27. La Rovere MT, Pinna GD, Raczak G (2008) Baroreflex sensitivity: measurement and clinical implications. Ann Noninvasive Electrocardiol 13(2):191-207

28. Jordan J, Tank J, Shannon JR, Diedrich A, Lipp A, Schroder C, Arnold G, Sharma AM, Biaggioni I, Robertson D, Luft FC (2002) Baroreflex buffering and susceptibility to vasoactive drugs. Circulation 105(12):1459-1464

29. Halliwill JR, Morgan BJ, Charkoudian N (2003) Peripheral chemoreflex and baroreflex interactions in cardiovascular regulation in humans. J Physiol 552(Pt 1):295-302

30. Hunt BE, Tamisier R, Gilmartin GS, Curley M, Anand A, Weiss JW (2008) Baroreflex responsiveness during ventilatory acclimatization in humans. Am J Physiol Heart Circ Physiol 295(4):H1794-1801

31. Simpson LL, Busch SA, Oliver SJ, Ainslie PN, Stembridge M, Steinback CD, Moore JP (2019) Baroreflex control of sympathetic vasomotor activity and resting arterial pressure at high altitude: insight from Lowlanders and Sherpa. J Physiol. 597(9):2379-2390

32. Eckberg DL, Bastow H 3rd, Scruby AE (1982) Modulation of human sinus node function by systemic hypoxia. J Appl Physiol Respir Environ Exerc Physiol 52(3):570-577

33. Limberg JK, Johnson BD, Holbein WW, Ranadive SM, Mozer MT, Joyner MJ (2016) Interindividual variability in the dosespecific effect of dopamine on carotid chemoreceptor sensitivity to hypoxia. J Appl Physiol 120(2):138-147

34. Jordan J, Toka HR, Heusser K, Toka O, Shannon JR, Tank J, Diedrich A, Stabroth C, Stoffels M, Naraghi R, Oelkers W, Schuster H, Schobel HP, Haller H, Luft FC (2000) Severely impaired baroreflex-buffering in patients with monogenic hypertension and neurovascular contact. Circulation 102(21):2611-2618

35. Christou DD, Jones PP, Jordan J, Diedrich A, Robertson D, Seals DR (2005) Women have lower tonic autonomic support of arterial blood pressure and less effective baroreflex buffering than men. Circulation 111(4):494-498

36. Jones PP, Christou DD, Jordan J, Seals DR (2003) Baroreflex buffering is reduced with age in healthy men. Circulation 107(13):1770-1774

37. Blitzer ML, Lee SD, Creager MA (1996) Endothelium-derived nitric oxide mediates hypoxic vasodilation of resistance vessels in humans. Am J Physiol 271(3 Pt 2):H1182-1185

38. Grant TL, McGrath JC, O'Brien JW (1985) The influence of blood gases on alpha 1- and alpha 2-adrenoceptor-mediated pressor responses in the pithed rat. Br J Pharmacol 86(1):69-77

39. Tamisier R, Norman D, Anand A, Choi Y, Weiss JW (2004) Evidence of sustained forearm vasodilatation after brief isocapnic hypoxia. J Appl Physiol 96(5):1782-1787

40. Weisbrod CJ, Minson CT, Joyner MJ, Halliwill JR (2001) Effects of regional phentolamine on hypoxic vasodilatation in healthy humans. J Physiol 537(Pt 2):613-621

41. Niewinski P, Janczak D, Rucinski A, Tubek S, Engelman ZJ, Jazwiec P, Banasiak W, Sobotka PA, Hart EC, Paton JF, Ponikowski P (2014) Dissociation between blood pressure and heart rate response to hypoxia after bilateral carotid body removal in men with systolic heart failure. Exp Physiol 99(3):552-561

42. Heusser K, Thöne A, Lipp A, Menne J, Beige J, Reuter H, Hoffmann F, Halbach M, Eckert S, Wallbach M, Koziolek M, Haarmann H, Joyner MJ, Paton JFR, Diedrich A, Haller H, Jordan J, Tank J (2020) The efficacy of electrical baroreflex activation is independent of peripheral chemoreceptor modulation. Hypertension 75:257-264

43. Pfoh JR, Tymko MM, Abrosimova M, Boulet LM, Foster GE, Bain AR, Ainslie PN, Steinback CD, Bruce CD, Day TA 
(2016) Comparing and characterizing transient and steady-state tests of the peripheral chemoreflex in humans. Exp Physiol 101(3):432-447

44. Xie A, Skatrud JB, Puleo DS, Morgan BJ (2001) Exposure to hypoxia produces long-lasting sympathetic activation in humans. J Appl Physiol 91(4):1555-1562
45. Collins FS, Varmus H (2015) A new initiative on precision medicine. N Engl J Med. 372(9):793-795 